\title{
Correction to: Gene expression profiles of pro-inflammatory mediators in the conjunctiva of patients with epiblepharon
}

\author{
Bo Ram Kim ${ }^{1} \cdot$ Yuri Seo ${ }^{2} \cdot$ Hyun Jung Lee ${ }^{3} \cdot$ So-Hyang Chung ${ }^{4} \cdot$ Jin Sook Yoon ${ }^{1}$ (i)
}

Published online: 19 April 2021

๑) Springer-Verlag GmbH Germany, part of Springer Nature 2021

\section{Correction to: Graefe's Archive for Clinical and Experi- mental Ophthalmology}

https://doi.org/10.1007/s00417-021-05089-0

In the original publication, affiliations 1 and 4 are the same. Yuri Seo's affiliation should also be changed to Yongin Severance Hospital.

The correct presentation is given in this publication.

Publisher's note Springer Nature remains neutral with regard to jurisdictional claims in published maps and institutional affiliations.

The original article can be found online at https://doi.org/10.1007/ s00417-021-05089-0.

So-Hyang Chung

chungsh@catholic.ac.kr

$\triangle$ Jin Sook Yoon

yoonjs@yuhs.ac

1 Department of Ophthalmology, Severance Hospital, The Institute of Vision Research, Yonsei University College of Medicine, 50-1 Yonsei-ro, Seodaemun-gu, Seoul 03722, Republic of Korea

2 Department of Ophthalmology, Yongin Severance Hospital, Yonsei University College of Medicine, Yongin, Republic of Korea

3 Department of Biochemical Engineering, Seoil University, Seoul, Republic of Korea

4 Department of Ophthalmology and Visual Science, Catholic Institute for Visual Science, Seoul St. Mary's Hospital,

College of Medicine, The Catholic University of Korea, \#505

Banpo-Dong, Seocho-Gu, Seoul 137-040, Republic of Korea 